\title{
Critical diagnostic delay associated with unusual presentation of hepatocellular carcinoma (HCC) with orbital metastases: a case report
}

\author{
Daria M. Filippini ${ }^{1}$, Alessandro Di Federico ${ }^{1}$, Barbara Lenzi ${ }^{1}$, Elisabetta Nobili ${ }^{1}$, Stefano Brocchi ${ }^{2}$, \\ Deborah Malvi ${ }^{3}$, Giovanni Brandi ${ }^{1}$ \\ ${ }^{1}$ Department of Experimental, Diagnostic and Specialty Medicine, University of Bologna, Sant'Orsola-Malpighi Hospital, Bologna, Italy; ${ }^{2}$ Radiology \\ Unit, Department of Experimental, Diagnostic and Speciality Medicine, Sant'Orsola Hospital, University of Bologna, Bologna, Italy; ${ }^{3}$ Department \\ of Pathology, University of Bologna, Sant'Orsola-Malpighi Hospital, Bologna, Italy \\ Correspondence to: Dr. Alessandro Di Federico. Medical Oncology Unit, Sant'Orsola-Malpighi Hospital, Via Massarenti 13 - 40138 , Bologna, Italy. \\ Email: alessandr.difederico@studio.unibo.it.
}

\begin{abstract}
Orbital metastases are an extremely rare finding in patients with hepatocarcinoma (HCC), especially as its first presentation. Therefore, the risk of misdiagnosis is high, as well as that of drastic delays of the therapeutic algorithm. Here we report a 71-year-old man presenting with orbital metastases as the initial sign of HCC, whose initial misdiagnosis led to the impossibility to start life-saving cancer treatment. The patient's history has begun on March 2018 with a growing tumefaction of the right orbit initially treted with antibiotics and corticosteroids without benefit. Subsequently, a facial CT scan showed a voluminous right intra-orbital mass, eroding the orbital roof. Tissue biopsy documented well differentiated malignant epithelial tumor cells. Under the suspect of primitive lachrymal gland tumor, the patient was admitted to the head and neck Unit with surgical intent. However, a subsequent 18F-FDG-PET documented the presence of liver lesions and multiple sites of metastasis. A new biopsy, this time on liver nodules, was carried out and the diagnosis of HCC was finally made. Samples from the first biopsy were then reviewed and judged consistent with HCC metastasis. Unfortunately, the initial misdiagnosis resulted in a six-month delay of the start of a therapeutic approach. During that time, patient's general conditions got extremely worse, making him unable to afford an antiblastic treatment. The patient died three months after the definitive diagnosis. This case suggests that the presence of intraorbital lesion requires a multimodal approach starting from the initial presentation. Performing a complete staging since tumor's clinical onset is mandatory, preferably before carrying out a tissue biopsy. Even though HCC represents a rare cause of intraocular metastasis, it needs to be ruled out when an orbital mass is documented, as the short median survival and the frequently poor conditions of HCC patients make a timely diagnosis crucial.
\end{abstract}

Keywords: Hepatocellular carcinoma (HCC); metastasis; misdiagnosis; treatment-delay; orbital metastases

Submitted Nov 24, 2020. Accepted for publication Jan 08, 2021.

doi: 10.21037/apm-20-2331

View this article at: http://dx.doi.org/10.21037/apm-20-2331

\section{Introduction}

Hepatocellular carcinoma (HCC) represents one of the most common causes of tumor-related death worldwide (1). Distant metastasis mainly involves lungs and bones, the latter conferring a particularly poor prognosis, with a 1-year survival rate of $50 \%$ (2). Orbital metastases are extremely rare in patients with HCC, especially at the onset. For this reason, their presence frequently leads to misdiagnosis with high risk of critical treatment delays, considering the narrow therapeutic window of these patients. Here we report a 71-year-old man with HCC, whose initial misdiagnosis caused by the peculiar clinical presentation led 

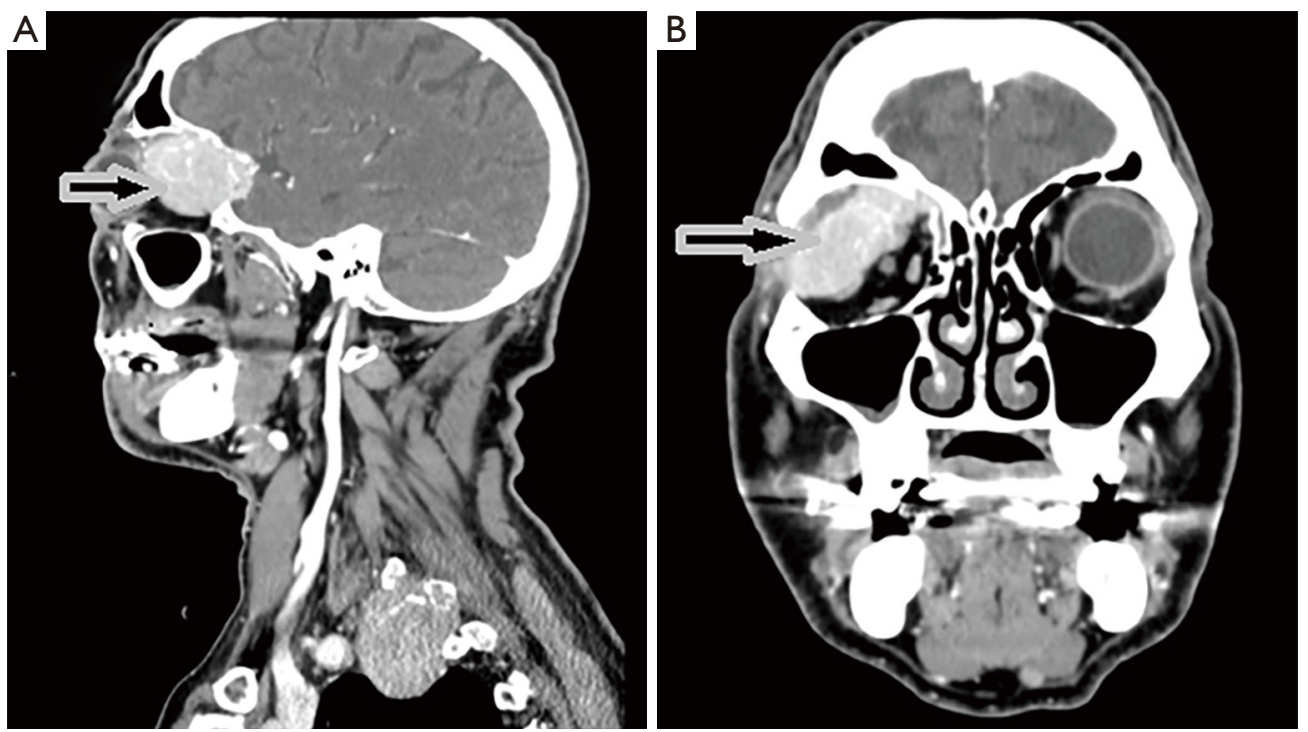

Figure 1 Sagittal (A) and coronal (B) CT scans of the large, intensely vascularized solid expanding process in the upper right portion of the right orbital cavity, associated with the lysis of the orbital roof and the infiltration of the surrounding tissues, including meninges, resulting in the compression of the cerebral parenchyma (arrows).

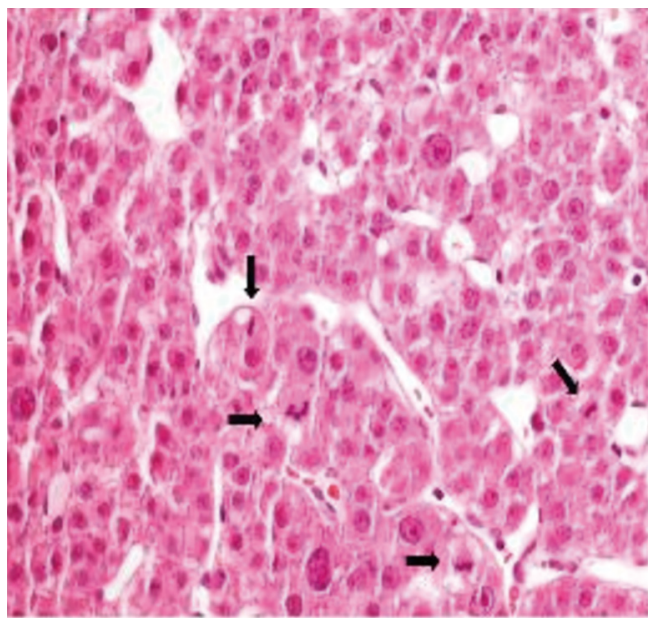

Figure 2 Tissue sample from the intra-orbital mass: the figure shows a malignant intensely vascularized epithelial neoplasm, with a nest and trabecular growth pattern. Moderate cytological atypia and a high mitotic index are also evident (arrows). Haematoxylin and eosin stain section, magnification $40 x$.

to the impossibility to start a correct anticancer therapy. We present the following article in accordance with the CARE reporting checklist (available at http://dx.doi.org/10.21037/ apm-20-2331).

\section{Case presentation}

The patient, a 71-year-old Caucasian man, with no underlying chronic liver disease, noticed a growing tumefaction of the right orbit on March 2018. He was visited by an ophthalmologist and initially treated with antibiotics and corticosteroids for a supposed diagnosis of an eyelid inflammation. The treatment was not effective, as the patient was hospitalized two months later due to the growth of the periorbital mass of the right eye, which caused pain, itching, ptosis, periorbital swelling and conjunctival erythema. Interestingly, in anamnesis, he reported an accidental serious exposure to glyphosate, a widely used herbicide, on his face and eyes, four months before the hospitalization. Even though we cannot affirm whether this event played a role on tumor genesis, glyphosate is a known hepatotoxic substance and its putative carcinogenic role is currently under debate. Neurological examination documented vertical diplopia and extraocular muscle deficit. Facial computed tomography (CT) scan showed voluminous intra-orbital mass, eroding the orbital roof (Figure 1). Biopsy using 18-gauge semi-automated needle (Figure 2) documented well differentiated malignant epithelial tumor cells, with variable size and prominent nucleoli, presumably originating from lacrimal glands, 

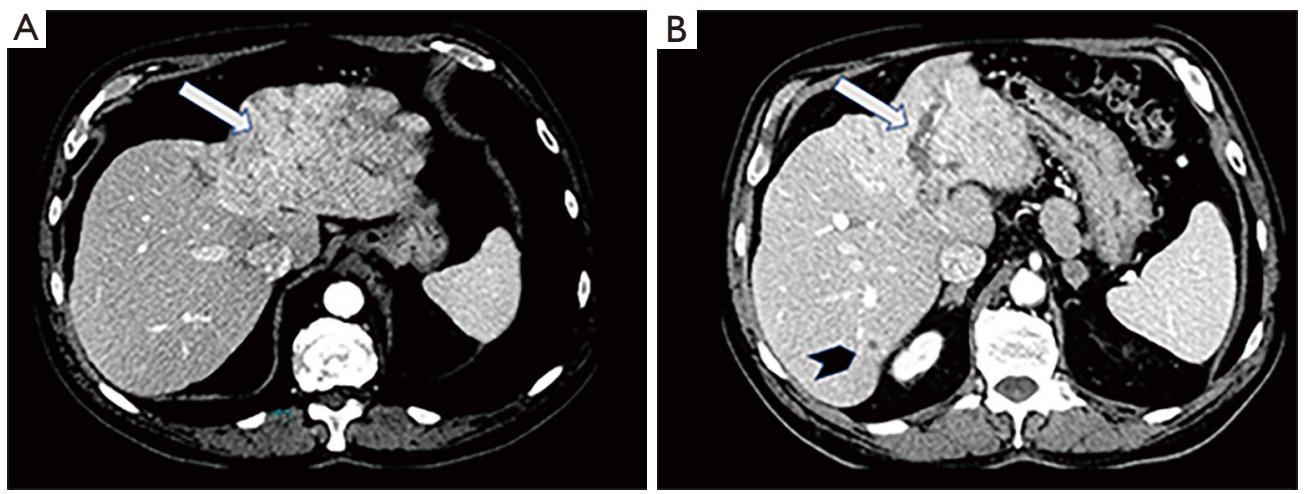

Figure 3 (A) Late arterial phase of a CT scan. The figure shows an infiltrative form of HCC (arrow). (B) late arterial phase of a CT scan. The figure shows a portal vein thrombosis (arrow) and a secondary lesion (arrowhead).

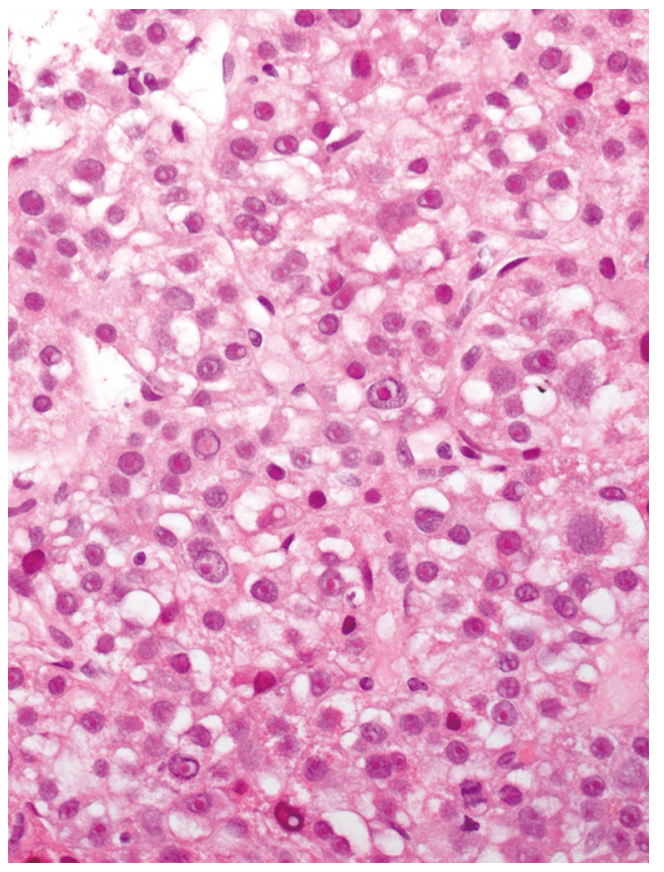

Figure 4 Liver biopsy (haematoxylin and eosin stain section, magnification 40x). The figure shows a hepatocellular carcinoma G3 according to Edmondson: loss of the normal hepatic architecture with a trabecular histological growth pattern is evident, as well as a marked nuclear pleomorphism with prominent nucleoli.

positive for CK8. Laboratory tests revealed mild elevation of aspartate aminotransferase (AST) (37 U/L; normal range $<35)$, lactic acid dehydrogenase $(\mathrm{LDH})(253 \mathrm{U} / \mathrm{L}$; normal range $<248)$ and alpha-fetoprotein $(31.2 \mathrm{ng} / \mathrm{mL})$, as well as the positivity for both anti-HBc and anti-
$\mathrm{HBe}$ antibodies. Quantitative hepatitis $\mathrm{C}$ virus (HCV) RNA reverse transcriptase polymerase chain reaction was performed on a serum sample and no viral RNA was detected. Hepatitis B virus (HBV) testing by quantitative polymerase chain reaction was also negative. The patient was then admitted to Head and Neck Surgery Unit of our Hospital with surgical intent, based on the hypothesis of lacrimal gland tumor. 18F-FDG-PET showed neoplastic tissue obstructing the upper airways and multiple metastases, involving lung, bone, adrenal glands and lymph nodes. At CT scan, a massive involvement of the left hepatic lobe of a lesion measuring $11 \mathrm{~cm}$ was documented associated to about three satellite lesions at the VI, IV and $\mathrm{V}$ segment, the largest of $2 \mathrm{~cm}$. It was an infiltrative form of HCC combined with a portal vein thrombosis. No clear sign of cirrhosis were noticed. Some abdominal lymph nodes were documented (Figure $3 A, B$ ).

Finally, on August 27, the patient was admitted to our Oncology Unit. Unfortunately, However, his clinical conditions got extremely worse over the prior three months, as he started suffering from dysphagia and required tracheostomy. On August 31, six months after the clinical onset and four months from hospitalization, liver biopsy (Figure 4) documented HCC, Edmondson G3, with macrotrabecular architecture (widespread immunoreactivity for HSA). The tumor was positive for human albumin gene. Mild hepatitis with septa was found in the extra-tumoral parenchima. Tumor samples from intra-orbital mass were then reviewed and deemed HCC metastases.

Considering the worsened performance status of the patient (ECOG PS 4), the impossibility to administer Sorafenib due to total dysphagia and the poor prognosis, only best supportive care could be offered. The patient died at the 
local hospice after three months from the definitive diagnosis.

All procedures performed in this study involving human participant were in accordance with the ethical standards of the institutional committee and with the Helsinki Declaration (as revised in 2013). Written consent for the use of data for scientific aims was obtained from patient's family. No written consent from patient was obtained because the patient is dead. The case report does not contain any information, besides anonymous medical data, that can be connected to the patient.

\section{Discussion}

Orbital metastases are unusual as first clinical presentation of neoplastic disease. Several case series reported lung, breast, melanoma and prostate cancer as the most frequent primary tumors $(3,4)$. In patients with HCC, the presence of orbital metastases represents a highly rare finding, and requires a multimodal approach involving expert ophthalmologists, surgeons, radiotherapists and oncologists starting from the initial presentation $(3,4)$. Moreover a detailed anamnesis should be done. Interestingly our patient had an accidental exposure on his face and eyes of glyphosate; even though we cannot affirm whether this event played a role on tumor genesis, glyphosate is a known hepatotoxic substance and its putative carcinogenic role is currently under debate.

A rapidly expanding orbital lesion infiltrating the surrounding tissues demands to perform a complete staging of the patient, with mandatory multidisciplinary approach starting from the initial presentation. In fact, in case of HCC as the primary tumor, the therapeutic window of these patients is extremely narrow, mainly because orbital metastasis strongly associated with very advanced disease and poor general conditions, with a median overall survival of about 10 months from the time of diagnosis, even using the most novel therapeutic approaches (5). The onset of anticancer treatment has been delayed due to the incomplete staging of disease that did not allow to identify the multiple hepatic lesions and the subsequent histopathological findings. Although HCC in non-cirrhotic liver often presents as a large solitary mass or dominant mass with peripheral satellite lesions, imaging features were not pathognomonic to identify primary HCC. The radiological diagnosis of primary hepatic malignancies in the non-cirrhotic liver is often difficult and histopathological analysis is necessary for a definitive diagnosis (6).
The presence of orbital metastasis of unknow primary tumor requires a multimodal approach starting from the initial presentation. Performing a complete staging since tumor's clinical onset is mandatory, preferably before carrying out a tissue biopsy. Even though HCC represents a rare cause of intraocular metastasis, it needs to be ruled out when an orbital mass is documented, as the short median survival and the frequently poor conditions of HCC patients make a timely diagnosis crucial.

In conclusion, HCC should be considered and ruled out among the possible causes of orbital masses. Timely diagnosis, complete radiologic disease staging and multidisciplinary approach are critical for these patients, as they often present very advanced disease with limited time to start an effective anticancer therapy.

\section{Acknowledgments}

Funding: None.

\section{Footnote}

Reporting Checklist: The authors have completed the CARE reporting checklist. Available at http://dx.doi.org/10.21037/ apm-20-2331

Conflicts of Interest: All authors have completed the ICMJE uniform disclosure form (available at http://dx.doi. org/10.21037/apm-20-2331). The authors have no conflicts of interest to declare.

Etbical Statement: The authors are accountable for all aspects of the work in ensuring that questions related to the accuracy or integrity of any part of the work are appropriately investigated and resolved. All procedures performed in this study involving human participant were in accordance with the ethical standards of the institutional committee and with the Helsinki Declaration (as revised in 2013). Written consent for the use of data for scientific aims was obtained from patient's family. No written consent from patient was obtained because the patient is dead. The case report does not contain any information, besides anonymous medical data, that can be connected to the patient.

Open Access Statement: This is an Open Access article distributed in accordance with the Creative Commons Attribution-NonCommercial-NoDerivs 4.0 International License (CC BY-NC-ND 4.0), which permits the non- 
commercial replication and distribution of the article with the strict proviso that no changes or edits are made and the original work is properly cited (including links to both the formal publication through the relevant DOI and the license). See: https://creativecommons.org/licenses/by-nc-nd/4.0/.

\section{References}

1. Montalto G, Cervello M, Giannitrapani L, et al. Epidemiology, risk factors, and natural history of hepatocellular carcinoma. Ann N Y Acad Sci 2002;963:13-20.

2. Kim S, Chun M, Wang H, et al. Bone metastasis from primary hepatocellular carcinoma: characteristics of soft tissue formation. Cancer Res Treat 2007;39:104-8.

Cite this article as: Filippini DM, Di Federico A, Lenzi B, Nobili E, Brocchi S, Malvi D, Brandi G. Critical diagnostic delay associated with unusual presentation of hepatocellular carcinoma (HCC) with orbital metastases: a case report. Ann Palliat Med 2021;10(7):8474-8478. doi: 10.21037/apm-20-2331
3. Chen CY, Zhong JH, Liu JL. Retrobulbar metastasis and intracranial invasion from postoperative hepatocellular carcinoma: A case report and review of the literature. Oncol Lett 2015;9:721-6.

4. Sochat MM, Piao J, Poddar N. Hepatocellular Carcinoma with Orbital Metastasis: a Unique Multidisciplinary Case Report. J Gastrointest Cancer 2019;50:978-82.

5. Eldesouky MA, Elbakary MA, Shalaby OE, Shareef MM. Orbital metastasis from hepatocellular carcinoma: report of 6 cases. Ophthalmic Plast Reconstr Surg 2014;30:e78-82.

6. Mamone G, Di Piazza A, Carollo V, et al. Imaging of primary malignant tumors in non-cirrhotic liver. Diagn Interv Imaging 2020;101:519-35. 